\title{
Systemic capillary leak syndrome after ChAdOx1 nCOV-19 (Oxford-AstraZeneca) vaccination
}

\author{
Julie Robichaud MD PhD, Catherine Côté BPharm MSc, Fanny Côté MD
}

- Cite as: CMAJ 2021 August 30;193:E1341-4. doi: 10.1503/cmaj.211212; early-released August 6, 2021

A 66-year-old man presented to the emergency department with a 1-day history of generalized malaise, nausea, abdominal pain and dizziness. On presentation the patient's body temperature was $36.5^{\circ} \mathrm{C}$, blood pressure $112 / 78 \mathrm{~mm} \mathrm{Hg}$, heart rate 112 beats/min and oxygen saturation 96\% (room air), and his respiratory rate was normal. He reported no allergies, no drug or alcohol misuse, and no current use of any medications or over-the-counter products. Two days earlier, he had received his first dose of the ChAdOx1 nCOV-19 (OxfordAstraZeneca) vaccine.

The patient's medical history included monoclonal gammopathy of uncertain significance (immunoglobulin $\mathrm{G}[\mathrm{IgG}] \mathrm{\kappa}$ ) and a cardiac arrest in 2017. At that time, he presented with generalized weakness and a syncopal episode. Because his hemoglobin level was elevated (210 [normal 130-180] g/L), polycythemia was suspected and phlebotomy was performed. Soon after, the patient became hypotensive and went into pulseless electrical activity arrest. He was successfully resuscitated, recovered without substantial deficits and was discharged home 5 weeks later. His test result for influenza type A was positive, and his shock was attributed to the viral infection.

At this presentation, the patient's hemoglobin level was increased markedly at $224 \mathrm{~g} / \mathrm{L}$. He had hypoalbuminemia (28 [normal 34-55] $\mathrm{g} / \mathrm{L}$ ) and an elevated creatinine level (133 [normal 62-115] $\mu \mathrm{mol} / \mathrm{L}$ ). Coagulation parameters, cardiac and liver enzymes, $\mathrm{C}$-reactive protein and procalcitonin were normal. Screening results for SARS-CoV-2 and extended respiratory virus panel were negative. Examinations using chest radiography, abdominal computed tomography, electrocardiography and focussed assessment with sonography for trauma echocardiography were unremarkable (Table 1 and Table 2).

We started intravenous fluids and empiric treatment with piperacillin-tazobactam, despite the unlikelihood of an infectious process. Twelve hours later, the patient had received more than $6 \mathrm{~L}$ of fluid, but his blood pressure had deteriorated to $93 / 60 \mathrm{~mm} \mathrm{Hg}$, his heart rate was 125 beats/min and his polycythemia persisted (hemoglobin $223 \mathrm{~g} / \mathrm{L}$ ). We admitted the patient to the intensive care unit (ICU). In the absence of other causes of impending shock, we diagnosed systemic capillary leak syndrome (SCLS).

\section{Key points}

- Rare cases of systemic capillary leak syndrome (SCLS) have been reported worldwide after vaccination with adenoviral vector and mRNA vaccines against SARS-CoV-2; a number have occurred in patients with a history of SCLS.

- Systemic capillary leak syndrome is a potentially lifethreatening immune disorder that is characterized by transient, recurrent episodes of vascular endothelial hyperpermeability that are often triggered by viral upper respiratory infections.

- Hypotension, hemoconcentration, hypoalbuminemia and anasarca are prominent features of SCLS, reflecting the massive leakage of fluids and proteins into peripheral tissues.

- Acute SCLS can rapidly evolve into shock and multiorgan failure, and treatment is mostly aimed at correcting hypovolemia and avoiding end-organ damage; prophylactic monthly administration of immunoglobulins can help prevent further episodes.

- Adenoviral vector vaccines against SARS-CoV-2 are not advised for patients with a history of SCLS.

During the first 24 hours of his admission, the patient received more than $10 \mathrm{~L}$ of intravenous fluid, but his hemoglobin and lactic acid levels remained elevated (Table 1), and his creatinine level continued to rise. Central venous pressure was consistently $0-1 \mathrm{~mm} \mathrm{Hg}$. He did not need vasopressors and he required oxygen therapy only transiently. He developed substantial anasarca and gained $15 \mathrm{~kg}$. Eventually, his hemodynamic status improved, laboratory abnormalities resolved, and he was discharged 4 days later.

We considered various causes of hypotension, polycythemia and hypoalbuminemia, and eliminated all of them (Table 2). Because his SCLS developed 2 days after vaccination against SARS-CoV-2 and we identified no other triggers, we suspected a possible adverse reaction to the ChAdOx $1 \mathrm{nCOV}-19$ vaccine and reported the reaction to our local department of public health.

\section{Interpretation}

Systemic capillary leak syndrome is a rare disorder associated with recurrent episodes of extravasation of fluid and protein into the interstitial space. ${ }^{1,2}$ Fewer than 500 cases have been 
Table 1: Laboratory test results for the patient during his 4-day stay in hospital

\begin{tabular}{|c|c|c|c|c|c|c|c|}
\hline \multirow[b]{2}{*}{ Laboratory test } & \multicolumn{7}{|c|}{ Test result } \\
\hline & $\begin{array}{c}\text { At } \\
\text { admission }\end{array}$ & $4 \mathrm{~h}$ & $12 \mathrm{~h}$ & $24 \mathrm{~h}$ & $48 \mathrm{~h}$ & $72 \mathrm{~h}$ & $96 \mathrm{~h}$ \\
\hline Hemoglobin, g/L (normal 130-180 g/L) & 224 & 226 & 223 & 184 & 142 & 131 & 136 \\
\hline Hematocrit, \% (normal 39\%-52\%) & 65 & 68.8 & 68 & 54.8 & 41.9 & 38.4 & 40 \\
\hline White blood cell count, $\times 10^{9} / \mathrm{L}$ (normal 4.4-11.0 $\times 10^{9} / \mathrm{L}$ ) & 14.5 & 19.7 & 24.5 & 21.5 & 11.4 & 7.6 & 6.9 \\
\hline Neutrophil count, $\times 10^{9} / \mathrm{L}$ (normal $1.8-7.0 \times 10^{9} / \mathrm{L}$ ) & 10.5 & 15.7 & 21.3 & 16.7 & 7.6 & 4.6 & 4.3 \\
\hline Lymphocyte count, $\times 10^{9} / \mathrm{L}$ (normal 1.0-4.0 × 109/L) & 2.4 & 2.2 & 2.4 & 3.2 & 2.5 & 2.1 & 2 \\
\hline Platelets, $\times 10^{9} / \mathrm{L}$ (normal $140-440 \times 10^{9} / \mathrm{L}$ ) & 222 & 237 & 251 & 202 & 173 & 164 & 174 \\
\hline Albumin, g/L (normal 34-55 g/L) & 28 & ND & ND & 25 & ND & ND & 38 \\
\hline Creatinine, $\mu \mathrm{mol} / \mathrm{L}$ (normal $62-115 \mu \mathrm{mol} / \mathrm{L}$ ) & 133 & 133 & 159 & 122 & 90 & 71 & 79 \\
\hline Lactate level, mmol/L (normal 0.5-2.2 mmol/L) & ND & ND & 3.8 & 3.3 & 1.1 & ND & ND \\
\hline D-dimer level, $\mu \mathrm{g} / \mathrm{L}$ (normal 0-500 $\mu \mathrm{g} / \mathrm{L}$ ) & 353 & ND & ND & ND & ND & ND & ND \\
\hline Prothrombin time, INR (normal 0.9-1.1) & 1.1 & ND & ND & ND & ND & ND & ND \\
\hline Partial prothrombin time, s (normal 20.1-26.4 s) & 28.4 & ND & ND & ND & ND & ND & ND \\
\hline
\end{tabular}

reported. Recognizing SCLS may be challenging, because presentation has often been preceded by a prodrome of flu-like symptoms and may be mistaken for sepsis. There are no specific diagnostic criteria for SCLS. Once other causes of shock have been excluded, the classical triad of hypotension, hemoconcentration and hypoalbuminemia supports the diagnosis of SCLS. ${ }^{2}$ Together with generalized edema, those 3 features are manifestations of the vascular hyperpermeability and extreme hypovolemia that occur with this syndrome.

The exact pathophysiology of SCLS is mostly unknown. Typically, exacerbations can be triggered by viral upper respiratory infections. ${ }^{1,3-5}$ An overwhelming immune response and upregulation of soluble inflammatory and angiogenic mediators during flares appear to be linked to vascular endothelial hyperpermeability. ${ }^{2}$ Monoclonal gammopathy of uncertain significance (predominantly $\lg \mathrm{K} \kappa$ ) is observed in $68 \%-85 \%$ of patients with SCLS, although a pathogenic role for the paraprotein has yet to be established. ${ }^{2}$ Reports exist of patients with SCLS who had a cardiac arrest triggered by influenza type A, similar to the experience of our patient in $2017 .{ }^{5,6}$ It is likely that his cardiac arrest at that time occurred during an unrecognized episode of SCLS; he had hypoalbuminemia ( $29 \mathrm{~g} / \mathrm{L}$ ), hemoconcentration and hypovolemia. However, SCLS was not suspected until this admission.

Our patient's near-fatal episodes illustrate that unrecognized SCLS can be life-threatening; SCLS is associated with an estimated 10-year mortality rate of 25\%-34\%. ${ }^{1,4}$ In addition to shock and renal and cardiopulmonary failure arising from intravascular volume depletion, thromboembolic events and compartment syndrome can occur. Systemic capillary leak syndrome can be classified as grade 1 (hypotension responding to oral hydration), grade 2 (intravenous fluids without hospital admission), grade 3 (life threatening and requiring admission to an ICU) and grade 4 (fatal). ${ }^{1}$
No interventions other than fluid resuscitation have been shown to halt or delay progression of a flare of SCLS. ${ }^{2}$ Most episodes are self-limited and resolve within 4 days. ${ }^{1}$ The frequency of recurrence of SCLS varies, ranging from once weekly to once every 10 years. Administration of prophylactic monthly intravenous Igs can reduce the frequency of episodes. ${ }^{1,2}$

The World Health Organization (WHO) reports that 3.8 billion doses of vaccines against SARS-CoV-2 have been administered worldwide (as of July 29, 2021; WHO Coronavirus [COVID-19] Dashboard, available at https://covid19.who.int). Adverse effects are usually mild and local in nature; however, rare serious adverse reactions can occur, such as pericarditis or myocarditis, anaphylaxis, Guillain-Barré syndrome and thromboembolic events with concurrent low platelet levels (the latter occurring mostly with adenoviral vector vaccines). ${ }^{7}$

In April 2021, the European Medicines Agency (EMA) reported 6 cases of SCLS following receipt of the ChAdOx1 nCOV-19 vaccine (including 1 fatality). ${ }^{8}$ Three of those patients had a previous history of SCLS. More than 78 million doses of the ChAdOx1 nCOV-19 vaccine have been administered in Europe, with a reported rate of 1 case of SCLS per 13 million doses. ${ }^{8}$ In June 2021, Health Canada issued the first report of SCLS in a patient who had received a ChAdOx1 nCOV-19 vaccine in Canada. ${ }^{9}$ The United Kingdom's Medicines \& Healthcare products Regulatory Agency (MHRA) reported 8 potential cases of SCLS that occurred shortly after administration of the ChAdOx1 nCOV-19 vaccine. ${ }^{7}$ Our patient had a score of at least 4 on the Naranjo Adverse Drug Reaction Probability Scale, ${ }^{10}$ making this a possible case of exacerbation of SCLS induced by the ChAdOx1 nCOV-19 vaccine.

Many health agencies have concluded that SCLS, albeit rare, should be considered a serious and potentially fatal adverse effect of the ChAdOx1 nCOV-19 vaccine, and are now advising against its use in patients with known SCLS. ${ }^{7-9}$ After the EMA's 
Table 2: Causes of polycythemia, hypoalbuminemia and hypotension and the reasons for exclusion in our patient

\section{Presentation}

\section{Polycythemia}

Primary polycythemia

Secondary

polycythemia

Relative polycythemia

\section{Hypoalbuminemia}

Hypotension and shock

\section{Differential diagnosis}

Reason for exclusion

\section{Inherited:}

Congenital heart defects

Acquired:

Polycythemia vera

Leukemia

Chronic hypoxia or cardiopulmonary abnormalities

Erythropoietin-secreting tumours

Dehydration Capillary leak syndrome Nephrotic syndrome

Poor nutrition or liver cirrhosis

Sepsis

Cardiogenic and obstructive shock

Anaphylaxis

Hereditary angioedema

Cancer drug reactions Hemophagocytic lymphohistiocytosis Viral hemorrhagic fever Snakebite envenomation
- Normal transthoracic echocardiogram

- Absence of Janus kinase 2 (JAK-2) mutation (Val671Phe)

- Absence of $B C R-A B L t(9 ; 22)$ mutation

- No clinical symptoms of obstructive sleep apnea and low risk according to the STOP-BANG score

- No known chronic pulmonary disease

- No obesity hypoventilation syndrome and no daytime hypercapnia

- No elevation of erythropoietin level: 4.6 (normal range 5.0-16.0) IU/L

- Normal hemoglobin and hematocrit levels between acute episodes

- No history of high-altitude travel

- Level of erythropoietin not high

- Not excluded

- No proteinuria, negative urine protein-to-creatinine ratio: undetectable (normal $<0.15 \mathrm{~g}$ protein/g creatinine)

- No evidence of hyperlipidemia that may be associated with nephrotic syndrome (normal fasting lipid profile)

- Rapid correction of serum albumin levels is inconsistent with hypoalbuminemia from poor nutrition or cirrhosis

- No features of liver cirrhosis or ascites on abdominal CT

- Normal procalcitonin level 0.28 (normal 0.00-0.39) $\mu \mathrm{g} / \mathrm{L}$ and C-reactive protein level 4.9 (normal $0.0-10.0) \mathrm{mg} / \mathrm{L}$

- No evidence of infection on chest radiography and abdominal CT

- Two negative results for blood cultures

- Negative result for urine culture

- Negative result for screening (RT-PCR) test for SARS-CoV-2 (COVID-19 direct diagnostic kit)

- Negative result for serology screening test for SARS-CoV-2 IgG

- Negative results for screening for common respiratory viruses and pathogens: syncytial respiratory virus; influenza type $\mathrm{A}$ ( $\mathrm{H} 1$ and $\mathrm{H} 3$ ); influenza type $\mathrm{B}$; parainfluenza viruses $1,2,3$ and 4; human metapneumovirus; entero-rhinovirus, coronavirus NL 63, HKU1, OC43 and 229E; adenovirus; Bordetella pertussis and parapertussis; Chlamydophilia pneumoniae; or Mycoplasma pneumoniae

- Negative results for serology screening test for HIV-1 and -2 (p24 combo)

- Normal FAST ultrasonography in the emergency department

- Normal electrocardiogram

- Normal transthoracic echocardiography and negative result for exercise stress test (recent outpatient investigations)

- Absence of pulmonary edema and raised central venous pressure

- Normal cardiac enzymes (troponin < 3 [normal 0-18] ng/L) and D-dimer levels (353 [normal $<500] \mu \mathrm{g} / \mathrm{L})$

- Negative V/Q

- Absence of typical clinical signs or symptoms associated with anaphylaxis (e.g., respiratory compromise, rash, pruritus, angioedema or persistent gastrointestinal symptoms)

- Normal tryptase level: 3.0 (normal < 11) $\mu \mathrm{g} / \mathrm{L}$

Normal C4 esterase activity $(0.20$ [normal $0.13-0.40] \mathrm{g} / \mathrm{L})$ and $\mathrm{C} 1$ esterase activity $(0.97$ [normal $0.69-1.42] \mathrm{g} / \mathrm{L})$

Absence of the epidemiologic factors usually associated with these diagnoses

\section{Other diseas
excluded}

Note: $\mathrm{CT}=$ computed tomography, FAST = focussed assessment with sonography for trauma, IgG = immunoglobulin G, RT-PCR = reverse transcription-polymerase chain reaction. 
July 2021 report of 3 cases of severe SCLS, 2 with fatal outcomes, which were potentially linked to the Ad26.COV2.S vaccine (Johnson \& Johnson-Janssen; an adenoviral vector vaccine), similar advice against using the Ad26.COV2.S vaccine in patients with a history of SCLS was made. ${ }^{8}$

In June 2021, a case series described 3 patients who presented with acute SCLS within 2 days of receiving a SARS-CoV-2 vaccine. ${ }^{11}$ They all had monoclonal gammopathy of uncertain significance and pre-existing SCLS. The 3 patients received different vaccines: Ad26.COV2.S, mRNA-1273 (Moderna) and BNT162b2 (Pfizer-BioNTech). To our knowledge, no other cases of SCLS have been reported that were associated with mRNA SARS-CoV-2 vaccines, and thus far no warnings or recommendations related to SCLS have been released regarding the MRNA1273 or BNT162b2 vaccines. Whether exacerbations are triggered by adenoviral vectors themselves or by SARS-CoV-2 antigen (spike protein) remains to be determined.

Administration of mRNA SARS-CoV-2 vaccines can still be used in patients with a history of SCLS, if the benefits are considered to outweigh the risks. After vaccination, physicians and patients must be extra vigilant, because exacerbations can develop quickly and patients may require urgent medical assessment. In our opinion, patients should be monitored closely during the 7-10 days after vaccination with home surveillance of blood pressure, heart rate, weight and urine output; hemoglobin and albumin levels should be measured at least once or twice. We also suggest that prophylaxis with intravenous immunoglobulins be started before vaccination, if not already given monthly.

\section{Conclusion}

We presented the case of a patient with an acute exacerbation of SCLS after ChAdOx1 nCOV-19 vaccination. Early recognition and treatment of acute SCLS is crucial. When a patient presents with generalized edema, malaise, hypotension, hemoconcentration or hypoalbuminemia shortly after administration of a SARS-CoV-2 vaccine, regardless of which vaccine was administered, SCLS should be considered. A history of SCLS is now considered a contraindication to adenoviral vector vaccines against SARS-CoV-2. Health care professionals should report any suspected adverse reactions to SARS-CoV-2 vaccines to their public health agency.

\section{References}

1. Druey KM, Greipp PR. Narrative review: the systemic capillary leak syndrome. Ann Intern Med 2010;153:90-8.

2. Druey KM, Parikh SM. Idiopathic systemic capillary leak syndrome (Clarkson disease). J Allergy Clin Immunol 2017;140:663-70.

3. Sousa A, Len O, Escola-Verge L, et al. Influenza A virus infection is associated with systemic capillary leak syndrome: case report and systematic review of the literature. Antivir Ther 2016;21:181-3.

4. Gousseff M, Arnaud L, Lambert M, et al. The systemic capillary leak syndrome: a case series of 28 patients from a European registry. Ann Intern Med 2011;154: 464-71.
5. Ebdrup L, Druey KM, Druey K, et al. Severe capillary leak syndrome with cardiac arrest triggered by influenza virus infection. BMJ Case Rep 2018 Aug. 29; 2018:bcr2018226108. doi: 10.1136/bcr-2018-226108.

6. Lawrence JL, Hindi H. Capillary leak syndrome aggravated by influenza type A infection. Cureus 2018;10:e2554.

7. Coronavirus vaccine - weekly summary of Yellow Card reporting. London (UK): Medicines \& Healthcare products Regulatory Agency (MHRA); 2021. Available: https://assets.publishing.service.gov.uk/government/uploads/system/ uploads/ attachment_data/file/1005194/Coronavirus_vaccine_-_summary_ of_Yellow_Card_reporting_14.07.21_clean.pdf (accessed 2021 July 18).

8. Pharmacovigilance Risk Assessment Committee (PRAC). COVID-19 pandemic. Amsterdam (Netherlands): European Medicines Agency (EMA). Available: https://www.ema.europa.eu/ (accessed 2021 June 11).

9. Recalls and safety alerts: Health Canada issues label change on the AstraZeneca and COVISHIELD COVID-19 vaccines. Ottawa: Health Canada; 2021 June 29. Available: https://healthycanadians.gc.ca/recall-alert-rappel-avis/hc-sc/2021 /75389a-eng.php (accessed 2021 July 28).

10. Naranjo CA, Busto U, Sellers EM, et al. A method for estimating the probability of adverse drug reactions. Clin Pharmacol Ther 1981;30:239-45.

11. Matheny M, Maleque N, Channell N, et al. Severe exacerbations of systemic capillary leak syndrome after COVID-19 vaccination: a case series. Ann Intern Med 2021 Jun 15;L21-0250 [Epub ahead of print]. doi: 10.7326/L21-0250.

\section{Competing interests: None declared.}

This article has been peer reviewed.

The authors have obtained patient consent.

Affiliations: Departments of Critical Care Medicine and Internal Medicine (Robichaud), Pharmacy (C. Côté) and Internal Medicine (F. Côté), CIUSSS Saguenay-Lac-Saint-Jean, Chicoutimi, Que.

Contributors: All of the authors interpreted the data and participated equally in writing the manuscript, gave final approval of the version to be published and agreed to be accountable for all aspects of the work.

Content licence: This is an Open Access article distributed in accordance with the terms of the Creative Commons Attribution (CC BY-NCND 4.0) licence, which permits use, distribution and reproduction in any medium, provided that the original publication is properly cited, the use is noncommercial (i.e., research or educational use), and no modifications or adaptations are made. See: https://creativecommons.org/ licenses/by-nc-nd/4.0/

Correspondence to: Julie Robichaud, julie.robichaud.med@ssss.gouv.qc.ca
The section Cases presents brief case reports that convey clear, practical lessons. Preference is given to common presentations of important rare conditions, and important unusual presentations of common problems. Articles start with a case presentation (500 words maximum), and a discussion of the underlying condition follows (1000 words maximum). Visual elements (e.g., tables of the differential diagnosis, clinical features or diagnostic approach) are encouraged. Consent from patients for publication of their story is a necessity. See information for authors at www.cmaj.ca. 